\title{
2M2C-R2ED: Multi-Metric Cooperative Clustering Based Routing for Energy Efficient Data Dissemination in Green-VANETs
}

\author{
D. Chandramohan ${ }^{1} \cdot$ Ankur Dumka $^{2} \cdot$ L. Jayakumar ${ }^{3}$ (D)
}

Received: 27 November 2019 / Accepted: 22 July 2020 / Published online: 20 August 2020

(C) Springer Nature Singapore Pte Ltd. 2020

\begin{abstract}
The aim of this paper is to focus on energy dissemination for green vehicle transportation. In current scenario population of urban areas keeps increasing as the people move towards the cities in search of jobs and betterlife style. This leads to an increase in the number of vehicles on the road. But the transport network which is accessible to the citizens is less when compared to their demand. This situation results in more vehicles on the road. The result of which is one of the factors that aggravate the traffic congestion. It is identified the vehicle volume on road is $91.7 \%$ which increases the $\mathrm{CO} 2$ emission to $93.25 \%$ every month. 2M2C-R2ED shows its efficiency in identification and segregation of alternative route by IWS with an average frequency of $3.82 \%$. Traffic congestion occurs when the available transport resources are less when compared to the number of vehicles that share the resource. As the number of vehicles increases the resources become scarce and congestion is more. The demand for the resources is higher than the actual capacity of the roads and the streets. There are some circumstances that which will aggravate the traffic congestion. The circumstances can be the road condition (potholes, road repair), accidents and some natural calamities. There is a lot of research being done to predict the traffic and model it in order to find a solution that will make the condition better. But still it is an open issue. The accuracy of the predictions done is less.
\end{abstract}

Keywords Green vehicle ad-hoc network $($ GVANET) $\cdot$ Clustering $\cdot$ Collision $\cdot$ Optimization · Intelligent water drop optimization (IWD) $\cdot$ Intelligent transport system (ITS)

\section{Introduction}

The Co2 emission during this congestion is also high in that particular area which causes a lot of health hazards for the people on the road. The mobility of the motorized transport is projected and then the related modal split is computed.

L. Jayakumar

jkaylogu@gmail.com

D. Chandramohan

pdchandramohan@gmail.com

Ankur Dumka

ankurdumka2@gmail.com

1 Department of Computer Science and Engineering, Madanapalle Institute of Technology \& Science, Madanapalle, India

2 Department of Computer Science and Engineering, Women Institute of Technology, Dehradun, India

3 Department of Computer Science, Department of Computer Science and Engineering, Vel Tech Rangarajan Dr.Sagunthala R\&D Institute of Science and Technology, Chennai, Tamilnadu, India
Based on this aggregate approach the future vehicle volume on road will be $91.7 \%$ which increases the $\mathrm{CO} 2$ emission to 93.25\%. [1] This emission of $\mathrm{CO} 2$ increases in a particular area when there is a huge increase in traffic volume or may be due to congestion. In order to avoid the transport network should support traffic diversions, good quality of the road and proper communication between the vehicles to infrastructure, vehicle to vehicle and among the infrastructure [2].

Research on the broadcasting of Signal Countdown Messages (SCMs) to vehicles via Vehicular Ad Hoc Network (VANET) [3] technology has shown that it can reduce CO2 emissions and energy consumption; however, past studies have lacked consideration of car-following and vehicle gliding mode. In this paper [4], two green driving suggestion models, namely, the Maximize Throughput Model (MaxTM) and the Minimize Acceleration and Deceleration Model (MinADM), are proposed to minimize the $\mathrm{CO} 2$ emissions by considering real-time traffic information nearby the intersection [5]. The two proposed strategies are compared with an Open Traffic Light Control Model (OTLCM). The main facts this paper demonstrate are that traffic models lack consideration of car following, which would make 
Fig. 1 Disaster Monitoring and Evacuation to minimize the risk factor in GVANET

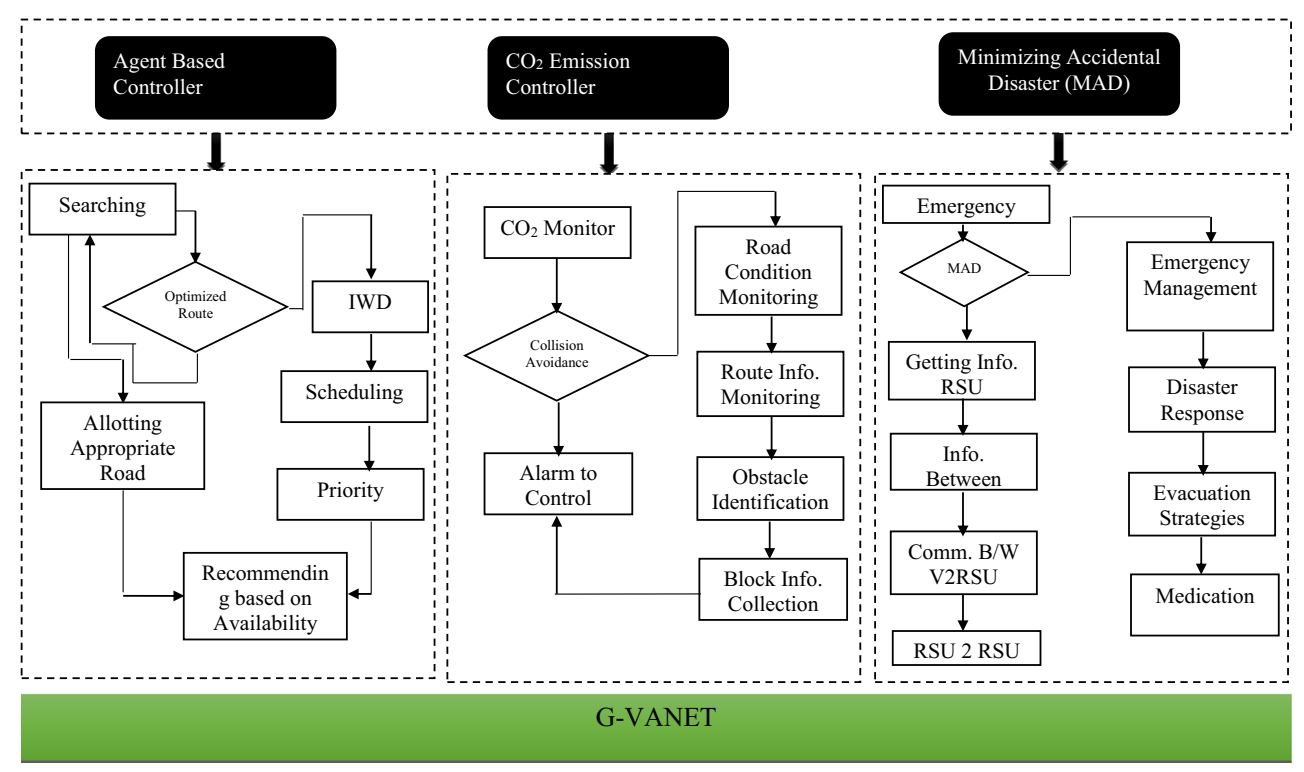

the simulation result unrealistic [6], that the proposed MaxTM can reduce more $\mathrm{CO} 2$ emissions than the MinADM and the OTLCM, and the total travel time in the MaxTM is also better than the other two models so that the general traffic performance can be improved [7]. Simulation results show that the performance of $\mathrm{CO} 2$ emissions of the MaxTM is $5 \%-102 \%$ better than the MinADM and 13\%-209\% better than the OTLCM in the simulation cases, and the performance of $\mathrm{CO} 2$ emissions of the MaxTM is $8 \%-14 \%$ better than the MinADM and $15 \%-$ $231 \%$ better than the OTLCM in the real traffic cases [8].

A computational model for flow and boundary shear stress fields in natural channels is combined with a bedload transport algorithm in order to investigate the genesis and finite-amplitude characteristics of riverine bars. [9] Since the time scales associated with bar growth and migration are typically much larger than the time scales of the flow, the flow is treated in a quasisteady manner, and the modification of the bed topography due to convergences and divergences in the sediment flux field is coupled to the flow model using a simple iterative technique [10, $11]$.

The topographic evolution algorithm yields accurate predictions of the development of point bars in curved channels and indicates that, while the origin of point bars is primarily due to curvature-induced secondary flow, the ultimate stability of these features is related to topographically-induced stream wise convective accelerations, as well as to

Fig. 2 Real-time monitoring of Moving object monitoring using GVANET

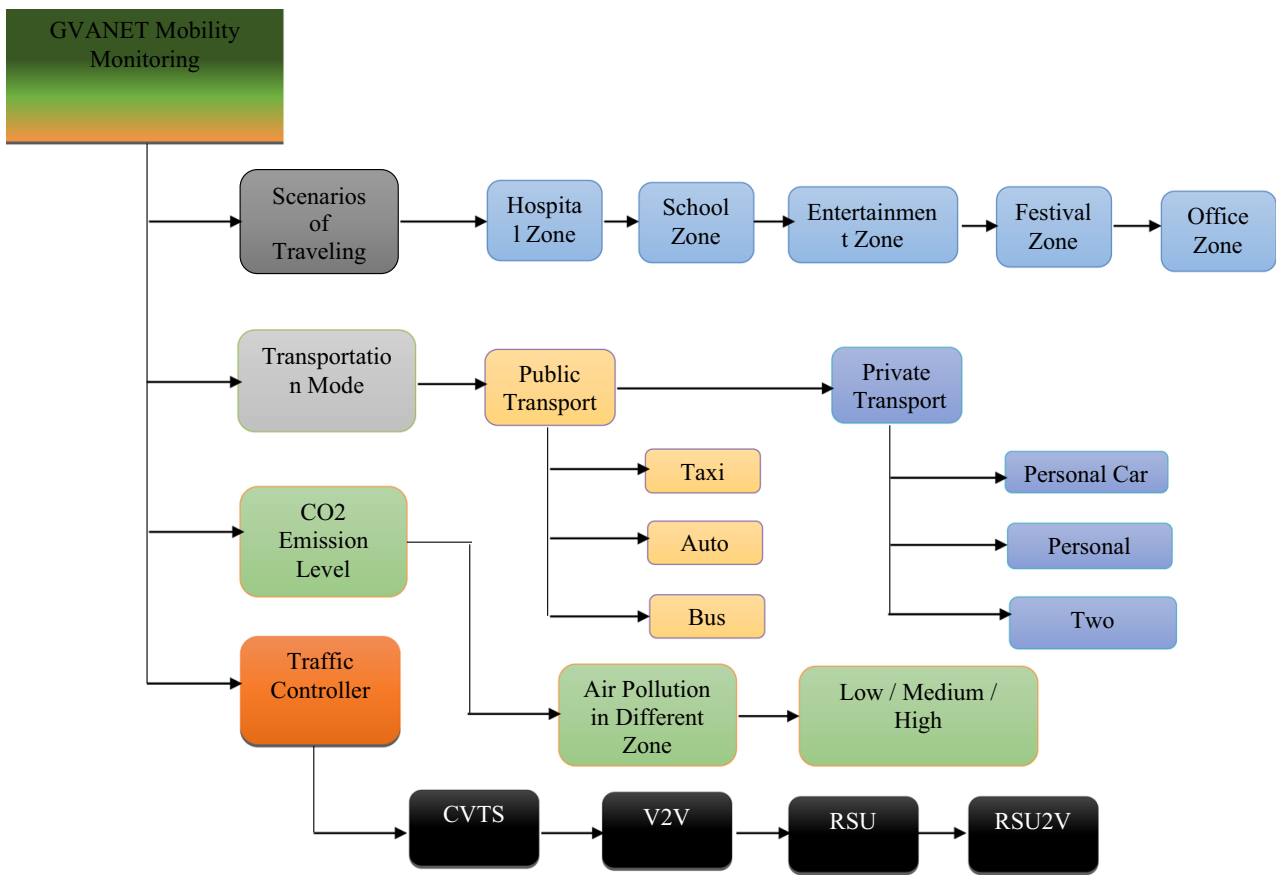


gravitational modification of sediment fluxes by bar slopes [12]. The technique presented herein is also used to investigate the mechanics of alternate bars in straight channels. A comparison of the nonlinear theory with a simple linear stability analysis for these features is used to demonstrate the importance of the nonlinear effects, and to provide a clearer physical understanding of the alternate bar instability [13].

Bhatia et al. [14] propose an artificial intelligent model for vehicular traffic behaviour monitoring as a data-driven approach by combining the flexibility, scalability, and adaptability of its activity. [15] A machine learning algorithm is developed as a long short-term memory neural network (LSTM-NN), which eliminates the back propagated error delay through memory block.

MusaBalta [16] developed a 3-stage fuzzy-decision tree model to monitor the traffic signalling, by focusing environmental factors and activity information like vehicle density vehicle signalling etc.,.[17]

Muhammad et al. [18], provide an extensive overview of the ITS and the evolution of ITS to VANETs discussed the privacy and security attacks in VANETs with their applications and challenges [19].
Sadia Din and Abdul [20], 5G-based SDN architecture for the utility of ITS. It have an adequate like sensing layer, relay layer, and core network.

Houssain et al., [21] an improvement of GPSR, named KFGPSR, where each vehicle estimate in real time the position of its neighbours by using kalman filter algorithm.

Houssain et al. an improved certificateless aggregate signature scheme without bilinear pairings for vehicular ad hoc networks Ismaila Adeniyi Kamil, Sunday Oyinlola Ogundoyin [22].

S.K. Lakshmanaprabu, [23] Big Data technologies to VANET to enhance traffic management process by planning and engineering. It also gains very useful insight from the massive quantity of operational data [24].

Malarvizhi et al. [25] ant colony optimization a fuzzy logic based is used for the optimization of IoV based services to model the heavy traffic. IoV based root selection method is compared for its effectives [26].

In contrast to point bars, the initial instability of alternate bars is shown to depend on simple topographic steering response [27], while the finite-amplitude characteristics of these features depend on gravitational effects and the production of
Fig. 3 Multi-metric Cooperative Clustering (2M2C) for GVANET Framework

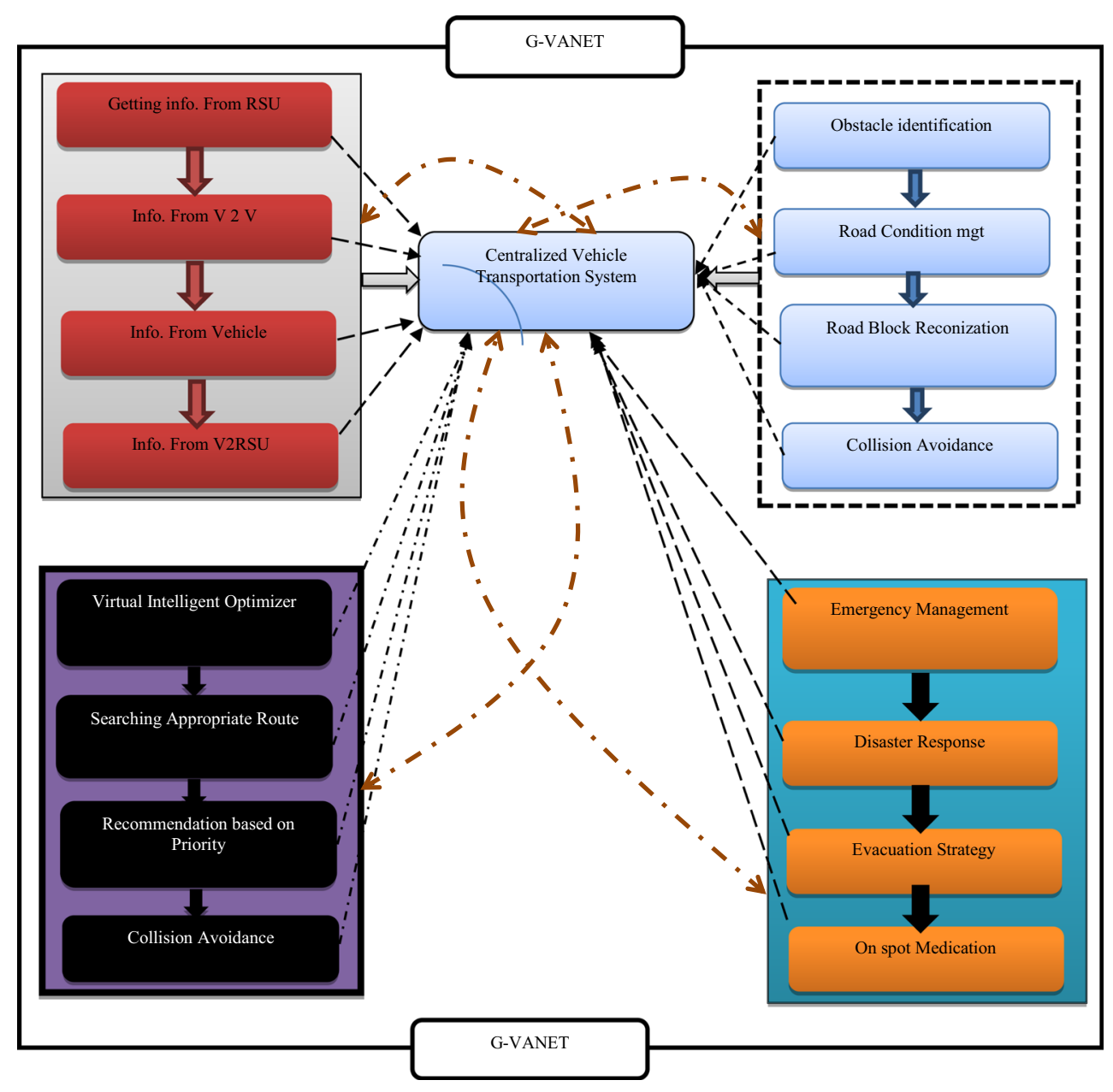


secondary flow associated with the curvature of flow streamlines. [28] The evolution model yields good predictions of the finite amplitude morphology of both point and alternate bars, as demonstrated by comparison of model predictions and measured bathymetry for several experimental studies [29].

\section{Review on Energy Efficient Data Dissemination}

Rodolfo Meneguette et al., inter vehicle communication in urban and high way traffic regions gets increasing drastically for controlling the traffic congestion and assisting to reduce the overflow of emission. An intelligent transportation system could control and reduces the fuel consumption, taking congested roads, trip time and in particularly the $\mathrm{CO} 2$ emission in vehicle transportation [14].

Ronald T. van Katwijk et al., to control the intersection of vehicles and road side unit communication for a possible vehicle based estimation to reduce the overall emission of vehicles. It acts as an advanced form of traffic adaptive control model for a predictive analysis of vehicle transportation. Moreover, it would control the acceleration and deceleration of vehicles by interacting with the infrastructure units deployed at road sides. It pay the way of benefiting in sinking the total time of travel for the general public in transit [16].

Yanjie Ji et al., finds the Pareto solution using genetic algorithm and microscopic emission model [18] that reduces the pedestrians and vehicles getting delay due to massive traffic. The multi-objective behavior of algorithm monitors the real world traffic data and controls the traffic signal for the betterment of vehicle drivers and the public. It appropriately reduces the impact on $\mathrm{CO} 2$ emission and increases the coordination of traffic signals which helps the public for tension free transportation.

Allan Mariano de Souza et al., an intelligent transportation system could reduce the economic loss of a country and decrease the drivers stress in traffic. ITS system monitors the greenhouse gas emission in both urban and rural regions. It supports the intra and inter vehicle communication process in controlling the traffic and pay a way to reduce the $\mathrm{CO} 2$ emission.

Yaonan Wang et al., [20] a bio-inspired approach and emission control analysis is derived from dynamic

Table 1 Dynamic node adjustment according to current condition

\begin{tabular}{llll}
\hline Default & PROD-Random & TEST-Random & DEV-Random \\
\hline Routers & 0 of 8 & 0 of 0 & 0 of 0 \\
Switches & 0 of 1 & 0 of 0 & 0 of 0 \\
Servers & 5 of 21 & 0 of 0 & 0 of 0 \\
\hline
\end{tabular}

Table 2 Static node searching

\begin{tabular}{llll}
\hline Default & PROD-PRS & TEST- PRS & DEV-PRS \\
\hline Routers & MAX & $>=$ MIN & 0 of 0 \\
Switches & Min & $>0$ & 0 of 0 \\
Servers & Minmax & $<=$ N-1 & 0 of 0 \\
\hline
\end{tabular}

combined control map technique. It uses the auxiliary power unit to control the emission of vehicles and balances the potential conflicts of fuel consumption. The quantitative analysis of the catalyst and the cause for the emission is analyzed by optimized techniques for the improvement of less fuel consumption.

Chunxiao $\mathrm{Li}$ et al., a three tire structure to control the traffic light dynamically to reduce the stop and go time of vehicles. Tire one of the architecture if designed from the electronic toll data to monitor the smoothening of the travel in recommended speed. From the data the tire two would communicate with traffic light and transfer it via antennas to reset of the vehicles to update the current information. Traffic light control algorithm [30] is used at the tire three level for intersection of all two tire data and helps to minimize the $\mathrm{CO} 2$ emission of stop and go vehicles flow.

Amilcare Francesco Santamaria et al., noticed that the US and European standard have been working on road safety for several decades. A Safety Enhancement WAVE based protocol (SeAWave) is proposed for active safety system. To monitor the emission level and obstacles present on the way of travel is identified by city traffic management system and it also update all information about the road conditions by communicating with the vehicles and road side units.

Xin Cao et al., observed in their study at china the major challenge for controlling the $\mathrm{Co} 2$ emission of vehicles keeps increasing drastically. It would be more effective to minimize the consuming of energy in auto industry by alternate energy saving measures. They proposed the Compertz model with different senarios based on life cycle analysis with IPCC standards. From the experimental result it is identified that $\mathrm{CO} 2$ emission gets reduced gradually.

An event notification framework [31] proposes an event publishing and subscription notification. The event dissemination from the road side units are controlled by the service providers. The event dissemination is done in such a way that it maximizes the subscription and the same time it minimizes
Table 3 Static node identification by various PDS

\begin{tabular}{llll}
\hline Default & PROD-PDS & TEST- PDS & DEV-PDS \\
\hline Routers & 0 of 8 & 0 or 1 & $0>=$ min \\
Switches & 0 of 1 & $0<>0$ & $>1$ \\
Servers & 5 of 21 & $0<=$ MAX & $\mathrm{N}$ \\
\hline
\end{tabular}


Table 4 Dynamic node identification by various PDS techniques

\begin{tabular}{llll}
\hline Default & PROD-CS & TEST- CS & DEV-CS \\
\hline Routers & $\mathrm{N}$ & $\mathrm{N}-1$ & 0 \\
Switches & $\mathrm{N}+1$ & $\mathrm{~N}$ & $(\mathrm{n}+1) / 2$ \\
Servers & 5 of N & 0 & $\mathrm{n}-1$ \\
\hline
\end{tabular}

the cost of event disseminations. [28] The communication is done between the roadside units and the vehicles, but this paper does not address the communication between vehicle to vehicle communications [32].

Rathore et al. (2010) proposes a reliable and low delay MAC protocol. The protocol aims in restricting the contention period of the channel as the messages that have to be transmitted will have a very short life time [33]. The road side units are clustered in order to reuse the frequency in non-adjacent clusters. Frequency reusing reduces the time to access the channel and also increases the availability of the bandwidth [34].

Finding an optimal route for the vehicles is a significant challenge in order to avoid collisions (Mayank et al. 2016). Meta heuristic approach is used to find the optimal route. The destination is predicted and the optimized route is found by forming regions. This algorithm can be implemented where prediction for an object is required [26].

Clustering is an issue that has to be addressed in VANETs. This is important since the road side units have to disseminate the data to the vehicles and the vehicles should also contact the road side units for any information. [35] If clustering is not done there redundant data and the communication is also hampered by not having a clear request response pattern. Clustering can be done by the relative speed of the vehicles, direction and the connectivity that vehicles have with other vehicles (Kakkasageri and Manvi 2014) [36].

Mahdi et al. [37], proposed the Gaussian particle swarm optimization (GPSO) algorithm to determine the stability of a system by a chaotic map using Gaussian function [38]. Moreover, the seeking abilities of particles can be promoted by computation effectiveness without affecting the efficiency of the fuzzy controller. Yan Cao et al. [39], proposed the

Table 5 Callout point of various devices in HNS

\begin{tabular}{llll}
\hline Default & PROD-HNS & TEST-HNS & DEV-HNS \\
\hline Routers & 1 of $\mathrm{n}$ & 1 of $\mathrm{n}$ & $\mathrm{N}$ \\
Switches & 1 of $\mathrm{n}-1$ & $\mathrm{n}$ & $\mathrm{n}-1$ \\
Servers & 2 of $(\mathrm{n}+1)$ & $\mathrm{N}+1$ & $\mathrm{n}+1$ \\
\hline
\end{tabular}

Table 6 Differential Zones acceptable node frequency

\begin{tabular}{llll} 
Search Status & Steps until collision & Collision Check & $\begin{array}{l}\text { No. of. Attempts } \\
\text { to Retrieve }\end{array}$ \\
\hline Z1 & 492 & 432 & 924 \\
Z2 & 122 & 109 & 231 \\
Z3 & 384 & 232 & 616 \\
Z4 & 277 & 51 & 328 \\
Z5 & 338 & 288 & 626 \\
Z6 & 489 & 365 & 854 \\
Z7 & 104 & 57 & 161 \\
\hline
\end{tabular}

seagull optimization algorithm for optimal parameter identification of the proton exchange membrane fuel cell stacks [40].

Man-Wen Tian et al. [32], deer hunting optimization algorithm it is a new improved algorithm for optimal configuration and shows the economic aspect of the power plant maintenance [41].

\section{Multi-Metric Cooperative Clustering for G-VANET Framework (2M2C)}

Due to the increase in the vehicle traffic management is essential to ensure safety, reduced time in reaching the destination. The traffic management system should monitor the incidents that happen and prompt the users about the incidents. In Fig. 1 shows, it's collaborations with the highway departments to take necessary actions to improve the road safety and increase the transport system efficiency. The products are available, but the deficiency is in executing in real time because of lack of proper communication techniques.

The products available, fill be fixed at the road side and on the vehicles. But the protocols used for the communication is not well defined. Communication protocol should be defined in order for the entire system to work efficiently. Moreover, all the communication should be done using wireless medium in dynamically changing topology which introduces another dimension of the problem that has to be thought through and

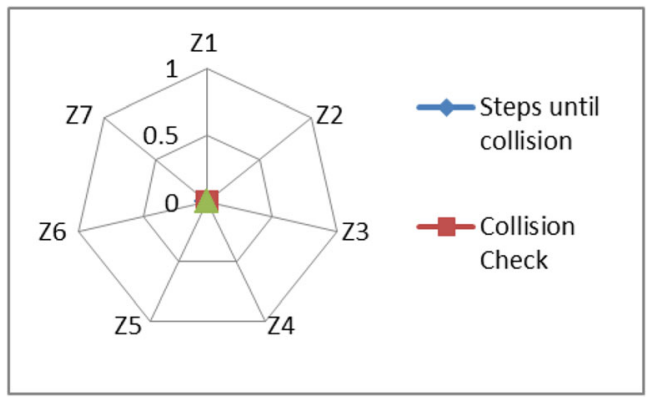

Fig. 4 Node identification with various frequencies under different network 
Table 7 Status of network nodes at random searching

\begin{tabular}{|c|c|c|c|c|c|c|c|c|}
\hline Random & Events & Alarms & Notifications & Outages & Surveillance & Heatmap & Distributed & Status \\
\hline $\mathrm{Z1}$ & 59 & 49 & 47 & 49 & 34 & 34 & 34 & 47 \\
\hline $\mathrm{Z} 2$ & 15 & 19 & 26 & 31 & 13 & 20 & 20 & 13 \\
\hline $\mathrm{Z3}$ & 21 & 16 & 12 & 13 & 20 & 13 & 13 & 12 \\
\hline $\mathrm{Z4}$ & 37 & 43 & 46 & 57 & 53 & 53 & 53 & 23 \\
\hline $\mathrm{Z5}$ & 53 & 34 & 43 & 53 & 43 & 57 & 34 & 57 \\
\hline Z6 & 26 & 13 & 19 & 20 & 13 & 37 & 13 & 13 \\
\hline $\mathrm{Z7}$ & 15 & 20 & 16 & 13 & 21 & 12 & 20 & 31 \\
\hline
\end{tabular}

analyzed. The wireless medium has a resource called as channels which have to be used efficiently as it a scarce resource.

In Fig. 2, the acquisition of data about the road quality, emergency, accidents and any other disasters should be processed by the road management system. The decision should be made and sent to the users in order to make them utilize the available transport resource efficiently. The data acquired by the infrastructure and the vehicles will be huge and will be redundant. The redundancy may cause confusion in taking the decisions and hence the data redundancy should be removed. There may be data which will not be used anywhere that may occupy a lot of space and a lot of bandwidth. These data should also be avoided during transmission from vehicle to infrastructure and vehicle to vehicle.

The other challenge encountered is data consistency and integration. Each device will give different types of data which will differ qualitatively and quantitatively. Hence the data should be analysed and data integrity should be maintained throughout the communication. The transport system developed should consider all these facts and then take a decision. The decision should provide a solution rather making the situation worse than the previous situation.

Proper communication protocol between:

- Infrastructure and vehicle

- Vehicle to vehicle
The goal is set to optimally utilize the channels which will reduce the interference. Interference cannot be completely eliminated since the frequency reuse will take place.

Figure 3 illustrates the efficient utilization of the channels which is a scarce resource of wireless communication. Optimal route allocation to avoid collision, congestion which eventually decreases the concentration of $\mathrm{CO} 2$. The operational cost is high which has to be reduced. Now the sensors which are manufactured are more powerful and the data collection is done efficiently. The cost of the computing devices is now reasonable and affordable which may even reduce the operational cost.

In the past few years the vehicles in an urban region increased terrifically in the global infrastructure. These peak deviations in utilization of vehicles evolution increase significant $\mathrm{CO} 2$ emission level and increase the air pollution. In VANET managing vehicles at peak traffic hour for controlling vehicle $\mathrm{CO} 2$ emission, road block identification, avoiding collision is the key issue during mobility. This issue raises the need of a scheduling mechanism for vehicles using the roads. An intelligent optimization approach could be adapted for searching the appropriate roots in real-time scenarios.

This proposal introduces the Intelligent Water Drop Optimization Algorithm (IWD) optimized route for vehicles in real time and scheduling the vehicle based on its priority. The system predicts the obstacles and blocks on the road by the Vehicle To Road Side Unit (V2RSU) communication system
Fig. 5 Random process to identify resources in different zones

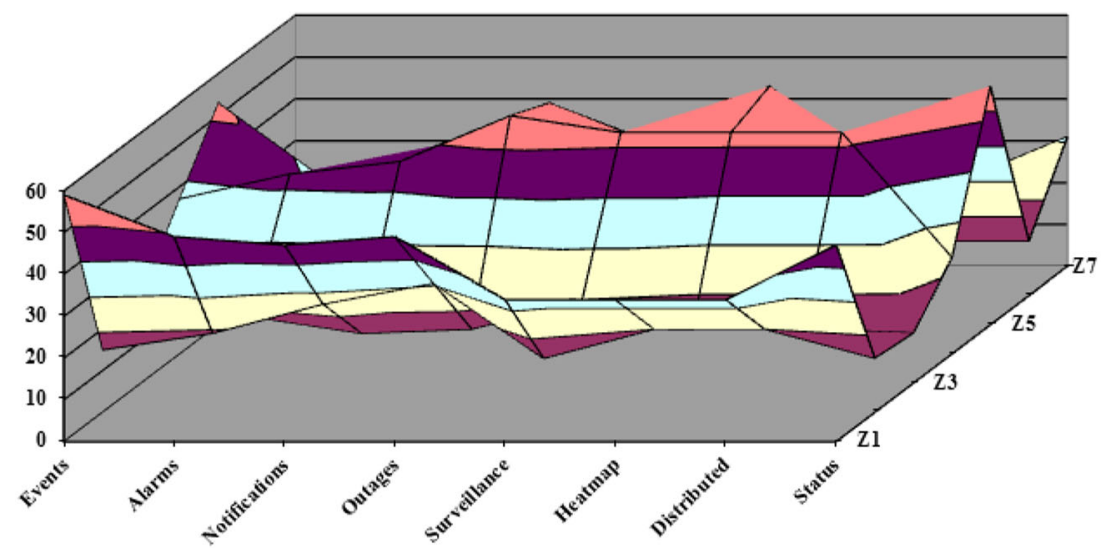


Table 8 Status of network nodes at pre-requesting search

\begin{tabular}{|c|c|c|c|c|c|c|c|c|}
\hline Pre-Req & Events & Alarms & Notifications & Outages & Surveillance & Heatmap & Distributed & Status \\
\hline Z1 & 49 & 47 & 46 & 43 & 43 & 54 & 53 & 47 \\
\hline $\mathrm{Z} 2$ & 16 & 12 & 12 & 19 & 19 & 37 & 30 & 46 \\
\hline $\mathrm{Z3}$ & 19 & 26 & 26 & 16 & 16 & 12 & 13 & 34 \\
\hline Z4 & 43 & 46 & 47 & 49 & 49 & 57 & 51 & 13 \\
\hline Z5 & 47 & 43 & 44 & 37 & 47 & 48 & 49 & 20 \\
\hline Z6 & 12 & 19 & 21 & 13 & 16 & 22 & 31 & 37 \\
\hline $\mathrm{Z7}$ & 26 & 16 & 13 & 23 & 25 & 17 & 13 & 12 \\
\hline
\end{tabular}

for predicting the best route for the user and giving priority to avoid the congestion. In real-time scenario the information from road side unit to road side unit, vehicle to vehicle and V2RSU are controlled by a centralized VANET managing system. The digression of alternative routes would initiate a reduction in $\mathrm{CO} 2$ emission globally in various zones and region. It consist of four different modules, in which to search the optimized route during peak hours rout assigning is based on priority and an appropriate route could be assigned to the user using IWD.

To identify the obstacles and blocks on the traveling region an intelligent transportation agent would be adopted by modified IWD algorithm for avoiding collision in VANET. This system also considers the emergency evacuation strategies as disaster identification for safe transportation. To provide BioVANET the $\mathrm{CO} 2$ emission could be reduced by monitoring the vehicle route selection, scheduling its rout, avoiding the vehicle collision and disaster recovering processes could be analyzed and processed using optimization approach. These issues are handled collaboratively using IWD and modified IWD algorithms to frame a Green VANET (GVANET). The vehicles at different zones which are running under various conditions are automatically controlled (engine changed to sleep mode) for energy saving in a heavy traffic situation. It could minimize the $\mathrm{CO} 2$ emission and control the air pollution for a GVANET scenario.

The information is collected about the obstacles, blocks, Co2 emission and any information related to emergency or disaster will be communicated by the devices fixed on the roads (Road side units) and the devices from the vehicles (vehicle units).

The communication can take place among the roadside units, road side unit and the vehicle unit or among vehicle units. The protocol that is developed for this communication should identify the roadblocks and other obstacles which will cause collision among the vehicles on the road. This congestion will also increase the $\mathrm{Co} 2$ level in that particular area.

GVANET Processing under various regions is described in finite time for dynamic vehicle communications in the vehicle transportation system. Vehicle to vehicle communication $(\mathrm{V} 2 \mathrm{~V})$, the intercommunication between vehicles could be represented with ' $\mathrm{t}$ ' time and the waiting time is represented as in Eq. (1).

$V_{2} V_{t} \rightarrow V_{2} V_{t-1}+\left(1-e^{\frac{-\left|V_{t}-V_{t-1}\right|}{\alpha}}\right)$

Vehicle to Road side unit communication process (V2R) is illustrated and the process is given by Eq. (2).

$V_{2} R_{t} \rightarrow V_{2} R_{t-1}+\left(1-e^{\frac{-\left|V_{t}-R_{t-1}\right|}{\delta}}\right)$

Roadside unit to the base station (R2B), for continuous updating of vehicle information is given as in Eq. (3).

Fig. 6 Source status of nodes at pre-requesting state

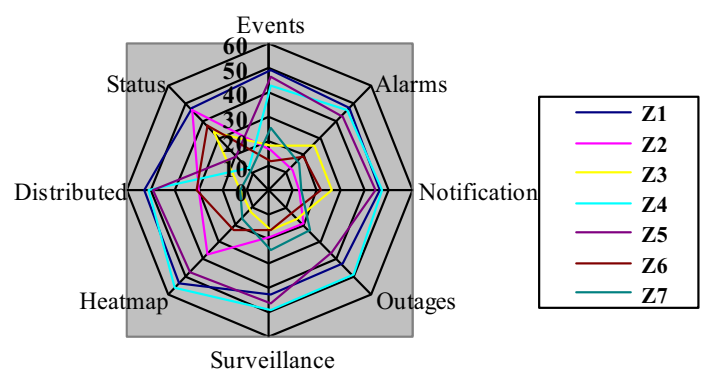


Table 9 Status of network nodes at post demand search

\begin{tabular}{|c|c|c|c|c|c|c|c|c|}
\hline Post Demand & Events & Alarms & Notifications & Outages & Surveillance & Heatmap & Distributed & Status \\
\hline $\mathrm{Z1}$ & 53 & 43 & 51 & 44 & 37 & 54 & 51 & 23 \\
\hline $\mathrm{Z} 2$ & 21 & 13 & 15 & 13 & 13 & 17 & 17 & 12 \\
\hline $\mathrm{Z3}$ & 30 & 13 & 26 & 21 & 23 & 26 & 22 & 13 \\
\hline Z4 & 51 & 44 & 47 & 43 & 56 & 53 & 48 & 47 \\
\hline $\mathrm{Z} 5$ & 57 & 54 & 46 & 53 & 37 & 54 & 54 & 43 \\
\hline Z6 & 15 & 12 & 12 & 23 & 20 & 17 & 12 & 13 \\
\hline $\mathrm{Z7}$ & 37 & 17 & 14 & 13 & 13 & 37 & 37 & 21 \\
\hline
\end{tabular}

$$
R_{t} B S_{t} \rightarrow R_{t} B S_{t-1}+\left(1-e^{\frac{-\left|R_{t}-B S_{t-1}\right|}{\alpha}}\right)
$$

Maximum travel speed of a vehicle is monitored by $\mathrm{V}_{\text {MAX }}$, and its process is denoted with respect to $V_{2} V_{t}<V_{M A X}$. To maintain the mobility frequency of a vehicle $V_{t}$ with respect to $\mathrm{t}-1$ times. $\left|V_{t}-V_{t-1}\right|$. If the signal strength gets varied in the Intelligent Vehicle Transportation system (IVT) the changes would be continuously be monitored by $\left(1-e^{\frac{-\left|R_{t}-B S_{t-1}\right|}{\alpha}}\right)$ and update the situations in Eq. (4).

$V_{2} V_{t}=V_{2} V_{T_{0}} \pm \int_{0}^{V_{M A X}}\left(1-e^{-\frac{v}{\alpha}}\right) d v$

The road side unit transformation $\mathrm{R}_{\mathrm{t}}$ with respect to $\mathrm{T}_{\mathrm{r}}$ and further identifications is noticed in $R_{t}<T_{r}(1-\varepsilon)$, Whereas ' $\mathrm{T}_{\mathrm{r}}$ ', representing the transmission range and to identify the ' $\alpha$ ', wireless channel condition is noticed using the equation [33].

$\alpha=V_{t} \cos \theta_{i}-V_{t} \cos \theta_{j}$

Base station (BS) has the information about all necessary transportation system to handle the intelligent rout calculation without affecting the regular activities of the vehicle distribution system BS and Vcom. The Eqs. (6) and (7) illustrate the BS information.

$$
\begin{aligned}
& B S=\left\{B S_{i}-B S_{j}\right\} \\
& V_{c o m}=V_{i} \sin _{\theta_{i}}-V_{j} \sin _{\theta_{j}}
\end{aligned}
$$
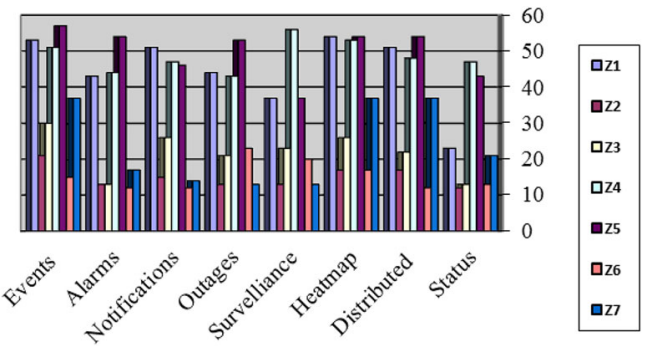

Fig. 7 Post demand search illustration
Vehicle communication $\left(\mathrm{V}_{\text {com }}\right)$ for GVANET system collaborates with base station, vehicle to vehicle, vehicle to road side units, road side units to base station. It would be a cyclic process for an effective transportation system. To customize the VTS for the betterment of emission less and to reduce collision, accidents, obstacle, emergency, disaster recovery, natural climatic changes, irregularity in traffic signal monitoring, etc. The intelligent transportation system (ITS) has been designed to control and fix the vehicle transfer issues. $R B=\left\{B S_{i}-B S_{j}\right\}$ Roadside unit to base station (BS) interprocess communication for a $\mathrm{V}_{\text {com }}$ light with a minimum route change is noticed and updated at using the Eq. (8).

$$
\left(R_{t} B S_{t}\right)_{n-1}=\min \left\{\left(L E T_{i}, \quad i+1\right),(i=1,2,3, \ldots, n)\right\}
$$

The automatic signal control system acts to be the initial eliminator for avoiding number of vehicles in high crowded regions $\min \left\{\left(L E T_{i, i+1}\right),(i=1,2,3, \ldots, n)\right\}$. However, it would not have sufficient time to control the flow of vehicles during notable peak hours. $\left(R_{t} B S_{t}\right)_{n-1}$ An intelligent decision making system would improve the betterment in controlling and directing to an alternate route without disturbing the normal activities. To reduce the $\mathrm{CO}_{2}$ emission an intellectual $\mathrm{V}_{\text {com }}$ process is embedded with collision avoidance and congestion control transportation system is incorporated using GVANET.

GVANET Intelligent Transformation (GVITi) is represented mathematically as in Eq. (9).

$G V_{I \mathrm{~T} i}=A l t\left(\frac{d\left(R V_{j}\right)}{R V_{i}}, \frac{d\left(R V_{k}\right)}{R V_{i}}+1\right)$

Where,

Alt Alternative route.

$R v_{j} \quad$ Vehicle selecting other route

$\mathrm{Rv}_{\mathrm{i}} \quad$ Vehicle at the spot having issue

$\mathrm{Rv}_{\mathrm{k}} \quad$ Vehicle got information to take decision

In unavoidable discrete finite state of vehicle route is defined by $\mathrm{V}_{\mathrm{i}}=\left\{\mathrm{V}_{\mathrm{i}}(\mathrm{R})\right\} \quad\{\mathrm{R}=0,1,2,3 \ldots \mathrm{n}\}$ as the Markova process of $V_{i}$ roots. Where $\{i \geq 0\}$ \& have a maximum roots $\mathrm{R}_{\max }$ ranges from current ' $\mathrm{C}$ ' position of Vehicles. 
Algorithm: Emission Reduction system in GVANET

Input:

Vi sense any obstacle or disturbance on the way of travel the current situation is intimated to upcoming vehicles $V_{j}$ which are yet to select the $V_{i}$ route.

Output:

To minimize the crowd at Vi proper information transmission metric $\operatorname{IT}_{M}$ is processed with $\operatorname{IT}_{M}$ where $m=\{0,1,2,3, \ldots n\}$

To reduce the vehicles $\left(\mathrm{V}_{\mathrm{i}}\right) \mathrm{CO} 2$ emission transported in various regions.

Start the GVANET info processing unit to do intelligent transportation system.

if $\left(\mathrm{V}_{\mathrm{j}}\right.$ have $\mathrm{IT}_{\mathrm{M}}$ gets low risk information $)$

Follow the same lane else it will select the alternated lane to reach the destination after updating the current status of the $V_{i}$ spot)

Else If $\left(\mathrm{V}_{\mathrm{j}}\right.$ receives any critical message from $\mathrm{IT}_{\mathrm{M}}$ through $\left.\mathrm{V}_{\mathrm{i}}\right)$

Do

$$
\text { GVANET processing unit }
$$

While $\left(\mathrm{V}_{\mathrm{i}}\right.$ reach the considerable safe route during transit)

End If

The scaled unit of ITM metric for each 'Mi' information transmission gets varied with it, where, it would vary from $0 \leq i \leq n$; do if $V_{i}[$ Source $]$ with no issue then it is assigned with positive transit road / way then

If (Positive Path)

$\mid$

$$
M_{i}=\left[\frac{V_{i}-V_{i \min }}{V_{\text {imax }}-V_{\text {imin }}}\right]
$$

Else if $\left(V_{i}[\right.$ Source $]$ is negative path)

$$
M_{i}=\left[\frac{V_{\text {imax }}-V_{i}}{V_{\text {imax }}-V_{\text {imin }}}\right]
$$

Else

The [Source] have the route of each vehicle $\mathrm{V}_{\mathrm{i}}$ passed by the same route.

$$
I T_{s}\left\{V_{i}, V_{j}\right\}=\sum_{i=1}^{\infty} M_{i}\left(R S U * V_{i}\right)
$$

End if

if (source of $\mathrm{V}_{\mathrm{i}}$ and $\mathrm{V}_{\mathrm{j}}$ with the maximum roots possibly selected as appropriate routes)

For $\left(V_{i}[\right.$ Source $] \& \& V_{j}[$ Source $]$ have the order of communication to each the Gateway of ITS)

if $\left(\left\{\left(V_{i}, V_{j}\right\} \geq\left\{V_{\text {imax }}, V_{\text {jmax }}\right\}\right)\right.$

RESET with alternative route in order to communicate with the $V_{j}$

New route for $V_{j}$ continues with $R V_{j}$

Repeat to avoid the collision and reduce the $\mathrm{CO}_{2}$ emission which gives an alert for living things under risk.

nd

End if

End for

End if. 
Table 10 A constrained based network node identification

\begin{tabular}{|c|c|c|c|c|c|c|c|c|}
\hline Constrained Zone & Events & Alarms & Notifications & Outages & Surveillance & Heatmap & Distributed & Status \\
\hline $\mathrm{Z1}$ & 54 & 37 & 51 & 53 & 31 & 44 & 30 & 12 \\
\hline $\mathrm{Z} 2$ & 17 & 15 & 49 & 34 & 57 & 44 & 13 & 46 \\
\hline $\mathrm{Z3}$ & 22 & 21 & 44 & 54 & 47 & 57 & 26 & 43 \\
\hline $\mathrm{Z4}$ & 48 & 59 & 53 & 49 & 56 & 57 & 21 & 23 \\
\hline $\mathrm{Z5}$ & 43 & 54 & 46 & 49 & 34 & 48 & 23 & 56 \\
\hline Z6 & 21 & 37 & 43 & 56 & 48 & 43 & 26 & 37 \\
\hline $\mathrm{Z7}$ & 13 & 12 & 59 & 54 & 57 & 46 & 21 & 53 \\
\hline
\end{tabular}

$\left\{\mathrm{R}_{\max } \geq C\right\}$ at ' $\left\{\tau_{f}\right\}$ ' seconds to reach the safe transit route as an alternative path. The algorithm below illustrates the process of reducing the $\mathrm{CO} 2$ emission for the proposed transport system.

The framework of the proposed project is given in Fig. 1. The data is collected in different areas and in different scenarios and analysed to take the decision by the centralized facility. The information is collected from the road side units and the moving vehicles. This information is used to study the current transport resources and the traffic condition. It is used to analyse the reason for the congestion. The factors that are responsible for the congestion may be any obstacle on the road, road quality, medical emergency and any natural disaster. The centralized facility will examine all the information and based on the conclusion of the analysis it will make a decision and forward it to the users. The protocol developed for the communication among the infrastructure, vehicle to infrastructure for this data communication will share a common medium. The wireless network uses the channels as the resources for communication. This channel should be properly utilized since bandwidth is the scarce resource in a wireless network. The channels are classified as overlapping channels and orthogonal channels. The overlapping channels cannot be used for communication since the signals will interfere with each other and hence the collision of the signal will happen. Orthogonal channels are used for communication as it does not interfere since the channels do not overlap. But the main challenge in using the orthogonal channels is the number of channels available for the communication. Orthogonal channels should be assigned to the wireless devices in such a way that it reduces the interference.

\section{Experimentation and Result Analysis}

It is illustrated in Table 1 for identification of ad-hoc nodes by PROD, TEST and DEV random searching process. However, if the nodes are identical in a static state it could be processed using PROD, TEST and DEV Pre-Requesting Search technique and it is shown in Table 2.

Similarly the static identification process verified with Post Demand Search techniques and it is illustrated in Table 3. The system encounters few constrained based identification of required information in limited period of time. Table 4 shows the process of searching the communicating nodes based on the limited information using PROD, TEST, and DEV constrained search technique. In Table 5 information call out is checked for its threshold and it's time to leave for searching the next immediate node for hand shake and to process the information with minimum dely. It is verified using PROD, TEST and DEV heterogeneous node searching process. The deviations are notices and illustrated in Tables 1, 2, 3, 4 and 5 respectively. Maximum wait time and number of participative nodes in various zones are critically $(n+1) / 2$ times. Optional nodes are identified $\mathrm{N}+1$ times with extend of $0<>$ MAX.

There would be amply of information in the nomadic computing process and the participative nodes with considerable energy to hand shaking with the crew. It have the process of verification until the nodes get collide each other. There
Fig. 8 Deviations of identical nodes using constrained resource

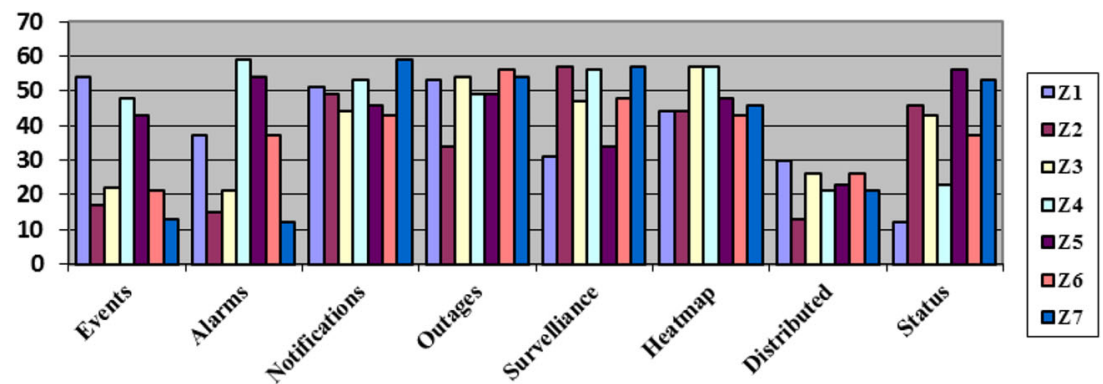


should be some collision check in all zones before starting the communication process and parallels the number of attempts to retrieve the original process by calculating it periodically. It is illustrated in Table 6 under various zones $Z_{1}, Z_{2}, Z_{3} \ldots Z_{7}$ is considered for this testing. Each and every zone is validated for collision check until it attempts to collide with other nodes. Various zonal collection checks are monitored and number of steps to handle the process also noticed for further tracking. Different network topology would be adopted in various zones $Z_{1}, Z_{2}, Z_{3} \ldots Z_{7}$ the collision check should be validated in all scenarios for the credit of cooperation in the topology. Figure 4 shows the deviations in searching the identical nodes in the communication process.

It could be filtered after the collision check gets completed. Similarly it retrieves the maximum number of attempts for a successful hand shake with neighbour nodes. The illustration of Table 6 shows the coincident of zones and its identical process in searching the needful process within stipulated time limit. Frequency range is taken into consideration for the limitation of collision process. The variations are calculated with respect to PROD, TEST and DEV searching and identification process under random, pre-request, post demand, constrained search and heterogeneous node searching techniques in all zones throughout the networking range.

If the requested sources are identified and its notable services are illustrated in Table 7. Contemporary nodes are focused by typical random searching process. The eccentricities are shown in Fig. 5 and its similarity eminence is fortified abruptly. Sporadic empathy takes place using pre-requesting state is illustrated in Table 8 and the deviations are noticed as shown in Fig. 6.

Events have its betterment in a zone, it triggers an alert with minimum range, and similarly is notified at different stages. Its outages, surveillance are distributed under various statuses. However, the nodes are pinpointed for its participation in a bewildering manifestation.

To find the misplaced and isolated post demand searching for identification of contemporary nodes which are grouped in

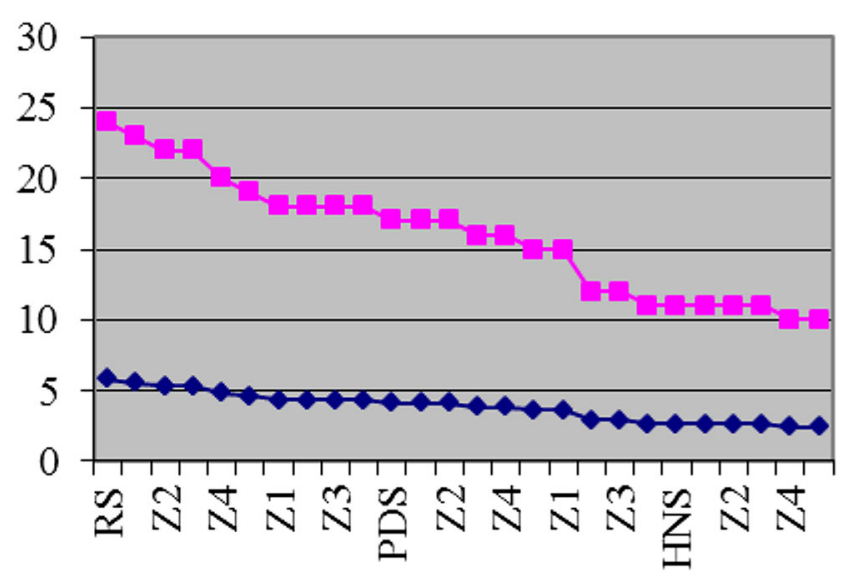

Fig. 9 Mobile computing devices authorization with various systems and approaches a typical searching quarantine is tabulated in Table 9 and its deviations are shown in Fig. 7. The constrained based identification process is illustrated in Table 10 and the periodic variations are shown in Fig. 8. From the illustration it is noticed that the status of searching process gets gradually minimize the search time and increase the sharing hindrance. Various event alarms are noticed with a limited surveillance and its status are distributed as plotted in Fig. 9.

In Table 11 the nomadic computing evaluations are illustrated and the iterations are noted under different zones from $\mathrm{Z} 1$ to $\mathrm{Z7}$ respectively. The simulation range is tested with $500 \times 500 \mathrm{~m}$ zone. It is identified form the result deviations tabulated under acceptable events and notifications at the time of conflicts. An

Table 11 Nomadic computing evaluation under various approaches

\begin{tabular}{|c|c|c|}
\hline Substring & $\begin{array}{l}\text { Request Frequency } \\
\text { node searching (in \%) }\end{array}$ & $\begin{array}{l}\text { No.of. Nodes } \\
\text { on Demand }\end{array}$ \\
\hline \multirow[t]{5}{*}{ RS } & 5.7971 & 24 \\
\hline & 5.5556 & 23 \\
\hline & 5.314 & 22 \\
\hline & 5.314 & 22 \\
\hline & 4.8309 & 20 \\
\hline \multirow[t]{5}{*}{ PRS } & 4.5894 & 19 \\
\hline & 4.3478 & 18 \\
\hline & 4.3478 & 18 \\
\hline & 4.3478 & 18 \\
\hline & 4.3478 & 18 \\
\hline \multirow[t]{5}{*}{ PDS } & 4.1063 & 17 \\
\hline & 4.1063 & 17 \\
\hline & 4.1063 & 17 \\
\hline & 3.8647 & 16 \\
\hline & 3.8647 & 16 \\
\hline \multirow[t]{5}{*}{$\mathrm{CS}$} & 3.6232 & 15 \\
\hline & 3.6232 & 15 \\
\hline & 2.8986 & 12 \\
\hline & 2.8986 & 12 \\
\hline & 2.657 & 11 \\
\hline \multirow[t]{6}{*}{ HNS } & 2.657 & 11 \\
\hline & 2.657 & 11 \\
\hline & 2.657 & 11 \\
\hline & 2.657 & 11 \\
\hline & 2.4155 & 10 \\
\hline & 2.4155 & 10 \\
\hline \multirow[t]{6}{*}{ 2M2C-R2ED_GVANET } & 2.8216 & 17 \\
\hline & 3.8236 & 25 \\
\hline & 4.1120 & 38 \\
\hline & 4.8123 & 45 \\
\hline & 4.9323 & 48 \\
\hline & 5.3145 & 54 \\
\hline
\end{tabular}

RS, Random Search; PRS, Pre-Requesting Search; PDS, Post Demand Search; $C S$, Constrained Search; HNS, Heterogeneous Node Search and proposed 2M2C-R2ED_GVANET approach 
Table 12 Identification of nodes by various approaches

\begin{tabular}{lll}
\hline Various approach & $\begin{array}{l}\text { Request frequency } \\
\text { node searching (in \%) }\end{array}$ & $\begin{array}{l}\text { Identified no.of. } \\
\text { nodes on demand }\end{array}$ \\
\hline RS & 4.8309 & 20 \\
PRS & 4.3478 & 18 \\
PDS & 3.8647 & 16 \\
CS & 2.657 & 11 \\
HNS & 2.4155 & 10 \\
2M2C-R2ED_GVANET & 2.8216 & 17 \\
\hline
\end{tabular}

alarm to make conscious about the situations and their current status is updated to the authorized users. The surveillance and heatmap distributions are noted and shown in Fig.9.

The described abbreviations are utilized in various figures and tables to find the substring variations under different frequency w.r.t percentages. Moreover, the demanded nodes are shortlisted based on the reachability and crisscross for its effectiveness as tabulated in Table 11.

\section{Discussion and Observation}

To identify various nodes on demand by various systematic approaches is illustrated in Table 12 with an average searching frequency $2.51 \%$.

The mobility of a system could be pragmatic in all traditional systems. It would be vicinity under plausible circumstances in communication era. In this paper the identification

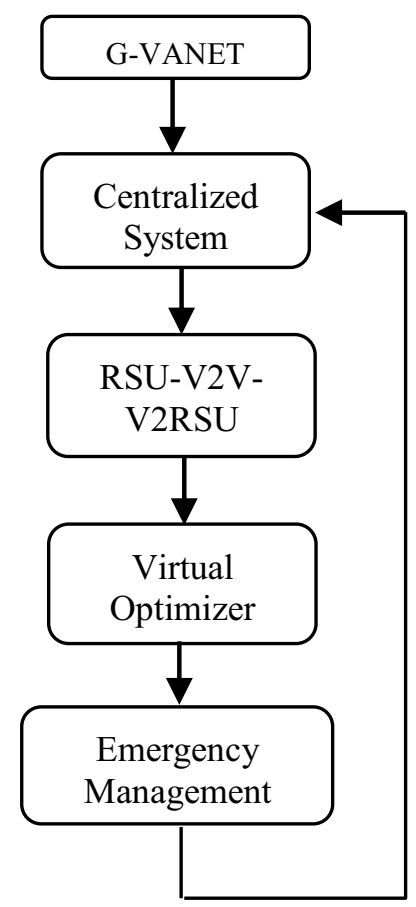

Fig. 10 Work flow for G-VANET of resources in a stipulated time to reduce the workflow in finding the impeccable conduit for an effective transmission. The frequency of empathy in searching the operative nodes under various scenarios and its substrings could be iterated using random search process. Moreover, pre-requesting search to find the uninterrupted sources and its corresponding nodes are possibly qualitied before it gets completion state. In post-demand search process would be calculated on demand request and regulated in a limited time (Fig. 10).

However, the cradles are sequestered with restricted number of energy necessities and coordinative treaties. The heterogeneous devices are communicative with possible outcome in all scenarios for identifying the appropriative source iterations.

\section{Conclusion}

In VANET to control the $\mathrm{CO} 2$ emission could be a challenging issue for reducing the pollution in urban region. ITS implemented with an automatic system for reducing the user interactions in vehicle transportation system. The urban vehicle population increases $\mathrm{CO} 2$ emission and pollutes the air which endangers the living regions into risky factors. However, IWD optimized algorithm is applied to eliminate the collision and having control on vehicle traffic system in urban regions. The proposed system shows an average of $2.8216 \%$ frequency for identifying various nodes and searching it for an effective process. It would improves the energy saving in VANET and helps for increasing the GVANET at polluted zones. The efficiency of GVANET is verified with existing ITS system, shows the proposed methodology have comparatively reduces the emission level in high traffic regions. The future enhancement might be focused on reducing the waiting time of vehicle at the source region of GVANET system.

\section{References}

1. Liu Kai, Ng Joseph K. Y, Lee Victor C.S., Son Sang H, Stojmenovic Ivan (2020) Cooperative data scheduling in hybrid vehicular Ad Hoc Networks (JUNE 2016) VANET as a software defined network. IEEE/ACM Transactions On Networking 24(3): 1759-1773

2. Fadda M, Murroni M, Popescu V (2016) Interference issues for VANET communications in the TVWS in urban environments. IEEE Transactions On Vehicular Technology 65(7):4952-4958

3. Fazio P, De Rango F, Sottile C (2016) A predictive cross-layered interference management in a multichannel MAC with reactive routing in VANET. IEEE Transactions On Mobile Computing 15(8):1850-1862

4. Ashok Kumar S, Chandramohan D (2018) Fault Test analysis in transmission lines throughout interfering synchrophasor signals. 
Elsevier- ICT Express 5:266-270. https://doi.org/10.1016/j.icte. 2018.03.003

5. Giang AT, Busson A, Lambert A, Gruyer D (2016) Spatial capacity of IEEE $802.11 \mathrm{p}$-Based VANET:models, simulations, and experimentations. IEEE Transactions On Vehicular Technology 65(8): 6454-6467

6. Gómez AA, Mecklenbräuker CF (2016) Dependability of decentralized congestion control for varying VANET density. IEEE Transactions On Vehicular Technology 65(11):9153-9167

7. Jiang X, Du DHC (2016) PTMAC: a prediction-based TDMA MAC protocol for reducing packet collisions in VANET. IEEE Transactions On Vehicular Technology 65(11):9209-9223

8. Chandramohan D, Rajaguru D, Vengattaram T, Dhavachelvan $P$ (2018) A coordinator specific privacy preserving model for e-health monitoring using artificial bee colony approach", John Wiley: Security and Privacy. (John Wiley) https://doi.org/10.1002/spy2.32

9. Kopp C, Tyson M, Pose R (2016) Tomographic reconstruction of RF propagation to aid VANET routing in urban canyons. IEEE Transactions On Vehicular Technology 65(12):9888-9901

10. Ubaidullah Rajput, Fizza Abbas, Heekuck Oh (2016) A hierarchical privacy preserving pseudonymous authentication protocol for VANET. IEEE ACCESS.pp.7770-7784

11. Cheng-Chi Lee, Fu Jen, Yan-Ming Lai and Pu-Jen Cheng, (2016) An efficient multiple session key establishment scheme for vanet group integration", IEEE Computer Society, pp.35-43

12. M.Shanmugam, L. Jayakumar, T. Anand, D. Rajaguru, Chandramohan Dhasarathan, J. Amudhavel (2018) Air pollution based vehicular routing problems: using genetic algorithm optimization approach", Foundation Environmental Protection \& Research-FEPR. Ekoloji: 27(106):e601. pp.1-13

13. Zhong H, Wen J, Zhang JCS (2016) Efficient conditional privacypreserving and authentication scheme for secure service provision in VANET. Tsinghua Science and Technology 21(6):620-629

14. Bhatia J, Dave R, Bhayani H, Tanwar S, Nayyar A (2019) SDNbased real-time urban traffic analysis in VANET environment. Comput Commun 149:162-175. https://doi.org/10.1016/j. comcom.2019.10.011

15. Ibrahim Q (2016) Event driven duty cycling: an efficient power management scheme for a solar-energy harvested road side unit. IET Electrical Systems in Transportation 6(3):222-235

16. MusaBalta İÖ (2020) A 3-stage fuzzy-decision tree model for traffic signal optimization in urban city via a SDN based VANET architecture. Future Generation Computer Systems Vol 104:142158

17. Abboud K, Zhuang W (2015) Impact of microscopic vehicle mobility on cluster-based routing overhead in VANETs. IEEE Transactions On Vehicular Technology 64(12):5493-5502

18. Muhammad A, Wang G, Bhuiyan MZA, Wang T, Chen J (2019) A survey on security attacks in VANETs: communication, applications and challenges. Vehicular Communications 19:100179

19. Akhtar N, Ergen SC, Ozkasap O (2016) Vehicle mobility and communication channel models for realistic and efficient highway VANET simulation. IEEE Trans Veh Technol:1-14. https://doi. org/10.1109/TVT.2014.2319107

20. Sadia Din AP, Abdul R (2018) 5G-enabled hierarchical architecture for software-Debned intelligent transportation system, computer networks. https://doi.org/10.1016/j.comnet.2018.11.035

21. Z.S. Houssain, I. Zaimi, M. Drissi, M. Oumsis, Saï El Alaoui Ouatik (2017) Trade-off between accuracy, cost and QoS using Beacon on Demand strategy and Kalman filtering over VANET, Digital Communications and Networks. https://oi.org/10.1016/j. dcan.2017.09.001

22. Alsabaan M, Alasmary W, Albasir A, Naik K (2016) Vehicular networks for a greener environment: a survey. IEEE Communications Surveys \& Tutorials:1-17. https://doi.org/10. 1109/SURV.2012.101912.00184
23. S.K. Lakshmanaprabu, K. Shankar, S. Sheeba Rani, Enas Abdulhay, Gustavo Ramirez, J. Uthayakumar, N. Arunkumar, "Sandeep Kumar Sood, Sahil, smart vehicular traffic management: an edge cloud centric IoT based framework, internet of things. Journal of Cleaner Production 217 (2019) 584e593

24. Chandramohan D, Vengattaraman T, Dhavachelvan P, Baskaran R, Venkatachalapathy VSK (2014)1350016) Fewss- framework to evaluate the service suitability and privacy in a distributed web service environment. Int. J. Model. Simul. Sci. Comput. (World Scientific) 5(1):1-37. https://doi.org/10.1142/ S1793962313500165 ISSN: 1793-9615

25. Malarvizhi, Kumar, Usha, Devi G, Gunasekaran Manogaran , Revathi Sundarasekar, Naveen Chilamkurti, R. Varatharajan (2018) Ant colony optimization algorithmwith Internet of Vehicles for Intelligent Trafbc Control System, Computer Networks, https://doi.org/10.1016/j.comnet.2018.07.001

26. Benslimane A, Taleb T, Sivaraj R (2011) Dynamic clustering-based adaptive mobile gateway management in integrated VANET - 3G heterogeneous wireless networks. IEEE Journal on Selected Areas IN Communications 29(3):559-570

27. X. Cao and Z. Wen (2015) CO2 emission reduction impacts of promoting energy-saving and new energy vehicles in China. 2015 Seventh Annual IEEE Green Technologies Conference, New Orleans, LA, pp. 110-116. https://doi.org/10.1109/ GREENTECH.2015

28. F. Santamaria, C. Sottile, A. Lupia and P. Raimondo (2014) An efficient traffic management protocol based on IEEE802.11p standard," International Symposium on Performance Evaluation of Computer and Telecommunication Systems (SPECTS 2014), Monterey, CA,, pp. 634-641. https://doi.org/10.1109/SPECTS. 2014.6880004

29. Li C, Shimamoto S (2012) An open traffic light control model for reducing vehicles hbox $\mathrm{CO} 2$ emissions based on ETC vehicles. IEEE Transactions on Vehicular Technology 61(1):97-110

30. de Souza AM, Villas LA (2015) A new solution based on intervehicle communication to reduce traffic jam in highway environment. IEEE Lat Am Trans 13(3):721-726. https://doi.org/10.1109/ TLA.2015.7069097

31. Joy CM, Gupta A, Sreenivas RC (2016) Event notification in VANET with capacitated roadside units. IEEE Trans Intell Transp Syst 17(7):1867-1879

32. Tian M-W, Yan S-R, Han S-Z, Nojavan S, Jermsittiparsert K, Razmjooy N (2020) New optimal design for a hybrid solar chimney, solid oxide electrolysis and fuel cell based on improved deer hunting optimization algorithm. Journal of Cleaner Production 249: 119414, ISSN 0959-6526. https://doi.org/10.1016/j.jclepro.2019. 119414

33. Wang Y, Shen Y, Yuan X, Yang Y (2015) Operating point optimization of auxiliary power unit based on dynamic combined cost map and particle swarm optimization. IEEE Trans Power Electron 30(12):7038-7050. https://doi.org/10.1109/TPEL.2014.2383443

34. Meneguette RI, Filho GPR, Guidoni DL, Pessin G, Villas LA, Ueyama J (2014) Increasing intelligence in inter-vehicle communications to reduce traffic congestions: experiments in urban and highway environments. PLOS ONE 11(8). https://doi.org/10. 1371/journal.pone. 0159110

35. van Katwijk RT (2014) Using I2V communication to reduce traffic emissions at urban signalized intersections. International Procedia Transport Research Arena-Social and Behavioural Sciences 48: 2385-2392

36. Yanjie Ji, Bo Hu Jing Han and Dounan Tang (2014) An improved algebraic method for transit signal priority scheme and its impact on traffic emission", International Journal for Mathematical Problems in Engineering", 11 pages. (Hindawi). https://doi.org/10.1155/ 2014/412132 
37. Mahdi, Mir, Mohammad, Dayyani, Tole, Sutikno, Morteza Mohammadi Zanjireh, Navid Razmjooy (2019) Employing a gaussian particle swarm optimization method for tuning multi input multi output-fuzzy system as an integrated controller of a microgrid with stability analysis. https://doi.org/10.1111/coin.12257

38. Chandramohan D, Manimaran A, Reddy R, Tripathi D (2019) Fog enabled secure and privacy obfuscation for IoT services. Jour.of Adv. Research in Dynamical \& Control Systems 11(Special Issue-08):1604-1610

39. Cao Y, Li Y, Zhang G, Jermsittiparsert K, Razmjooy N (2019) Experimental modeling of PEM fuel cells using a new improved seagull optimization algorithm. Energy Reports 5:1616-1625, ISSN 2352-4847. https://doi.org/10.1016/j.egyr.2019.11.013
40. Cheng S-T, Horng G-J, Chou C-L Using cellular automata to form car society in vehicular ad hoc networks. IEEE Transactions On Intelligent Transportation Systems 12(4):1374-1384, 2011

41. Jack Fernando Bravo-Torres, Martín López-Nores et al (2016) Optimising reactive routing over virtual nodes in VANETs", IEEE Transactions on Vehicular Technology.pp.1-22. https://doi. org/10.1109/TVT.2015.2426683

Publisher's Note Springer Nature remains neutral with regard to jurisdictional claims in published maps and institutional affiliations. 